\title{
Impact of environmental conditions on the reliability of MEMS components from optical applications
}

\author{
Marius Pustan ${ }^{1, *}$, Corina Birleanu ${ }^{1}$ and Florina Serdean ${ }^{1}$ \\ ${ }^{1}$ Micro \& Nano Systems Laboratory, Mechanical Systems Department, Technical University of Cluj- \\ Napoca, 400641 Muncii Street, Romania
}

\begin{abstract}
The reliability design and the lifetime of MEMS from optical applications is strongly dependent on the operating conditions. In optical devices an additional stress is introduced by the temperature gradient appearing during operation. The thermal stress introduced in the structure modifies the component response and its lifetime. The investigated sample is a micromirror fabricated from gold using the MEMS technologies and tested by an atomic force microscope. The scope is to analyse the temperature and relative humidity influence on the mechanical and tribological behaviours. Using a mechanical force given by the bending deflection and stiffness of the AFM probe, the dependence between the applied force and the micromirror deformation is monitored as a function of temperature. By increasing the temperature of structures the stiffness decreases based on the thermal relaxation of the material. If the relative humidity increases the adhesion between the micromirror and substrate is improved based on the capillarity effect. The results obtained are useful to MEMS designers who can predict the micromirror response as a function of the operating conditions with influence on the actuation energy, the accuracy in response and the system lifetime.
\end{abstract}

\section{Introduction}

Micro-opto-electro-mechanical Systems (MOEMS) integrate optical, mechanical and electrical components on a single chip and allow the use of various optical elements in a compact and low-cost device. Some principle commercial applications of MOEMS are: optical switches (for telecommunication routeing), displays (head up displays, vehicle displays), shutter arrays (switching), tuneable filters (broadband systems), waveguides (biomedical/ diagnostic), environmental pollution detection (multisensory arrays with optical detection) [1]. The main mechanical part of MOEMS is a moving component as microbridges, microcantilevers or a more complicated structure such as a micromembrane. These flexible components are integrated in MOEMS as mirrors, shutters, filters and attenuators [2]. The fabrication technology of MOEMS is based on micromachining

\footnotetext{
*Corresponding author: Marius.Pustan@omt.utcluj.ro
} 
techniques combined with integrated circuits (IC) processing methods. The integration of the components in a complex MOEMS device can be performed in a monolithically method or a hybrid one. The micromachining technology together with IC- based processing techniques is used in the fabrication of complex opto-electronic integrated circuits and micro-electromechanical alignment devices in production quantities [1].

The failure mechanism of Micro-electro-mechanical Systems (MEMS) is more complicated than in microelectronics. For instance, the MEMS devices interact with different environmental conditions such as temperature or humidity. The reliability design of MEMS must consider the environmental effect on the device response and the stresses that are induced by the environment [3]. The Physics of Failure approach is one method to improve the reliability of MEMS. This approach can be used to determine the effect of environmental stress of MEMS sensitivity [3].

In order to be able to perform experimental tests on MEMS components as a function of environmental conditions the testing equipment must be integrated into an environmental chamber with the possibility to change the temperature, the humidity levels and the medium pressure. A thermal gradient introduces softening due to Young's modulus - temperature relation and a thermal relaxation, which changes the rigidity of material $[4,5]$. The lifetime of optical MEMS devices can also be influenced by temperature. The temperature changes the stiffness of the investigated structure with influence on the restoring force of components from substrate after stiction. Stiction is a very common reliability problem in MEMS devices. Stiction occurs when the adhesion force between contacting surfaces exceeds the restoring force that stiffness is dependent. Adhesion depends on the capillary condensation, which is affected by the moisture and surface contamination as well as the van der Waals forces that are influenced by the surface roughness. The medium humidity increases the adhesion based on the capillary forces between contact surfaces.

The next sections present the experimental tests performed on a MEMS component from optical application fabricated from gold as a reflective material. The sample is experimentally investigated in an environmental chamber with the possibility of temperature control as well as the environmental relative humidity $(\mathrm{RH})$.

\section{Experimental procedure}

\subsection{Sample description}

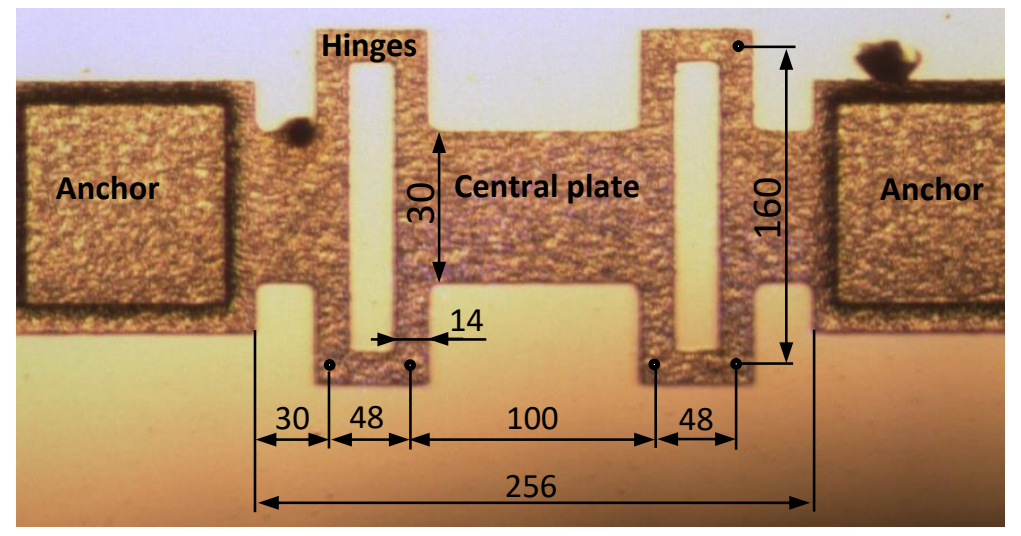

Fig. 1. Micromirror used in experimental investigations and the geometrical dimensions in $\mu \mathrm{m}$. 
The optical MEMS component used in the experimental investigation is a micromirror (Fig.1) fabricated using MEMS technology from a reflective material (gold) on a silicon substrate [4]. The gold material is the most used material in optical applications. The central plate of micromirror is supported by two rectangular hinges connected to the anchors. The rectangular hinges of structure are introduced in order to compensate the thermal expansion of the structure under the action of a thermal gradient. The central plate of structure can perform out-of-the plane motion. The thickness of flexible plate is $3 \mu \mathrm{m}$ and the gap between it and the silicon substrate is $2 \mu \mathrm{m}$. The others in plane geometrical dimensions of the flexible part (in $\mu \mathrm{m}$ ), measured by using an optical microscope, are presented in Fig.1.

\subsection{Atomic force microscopy procedure}

In order, to determine the environmental conditions effect on the micromirror behaviour, an Atomic Force Microscope (AFM) XE70 was used. The spectroscopy-in-point operating mode is used to deflect the sample from the initial position toward the substrate under different testing conditions (temperature and humidity). Using this AFM module, the vertical displacement of the scanning head together with the AFM probe is controlled by the AFM software. The AFM probe used in the experiment (TD21464) has a stiffness equal by $156 \mathrm{~N} / \mathrm{m}$ and the resonant frequency is $54 \mathrm{kHz}$. In order to investigate the temperatures influence on the mechanical response of the AFM probe, it vibrates close to the thermal stage and the resonant frequency is monitored during increasing temperature. The resonant frequency of $\mathrm{AFM}$ probe is not modified if the medium temperature increases from $20^{\circ} \mathrm{C}$ to $140^{\circ} \mathrm{C}$.

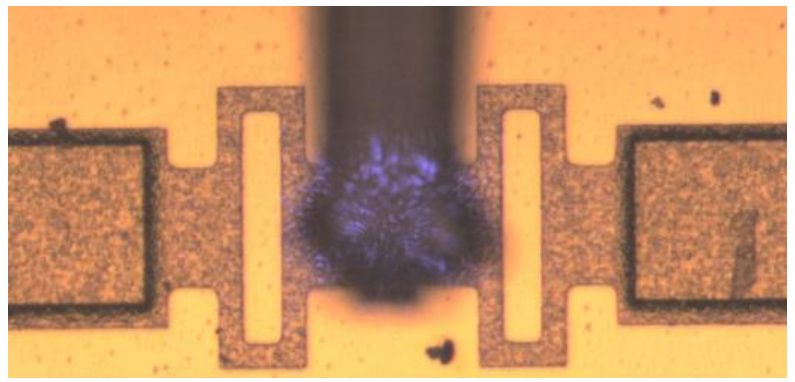

Fig. 2. AFM probe in contact with the investigated micromirror.

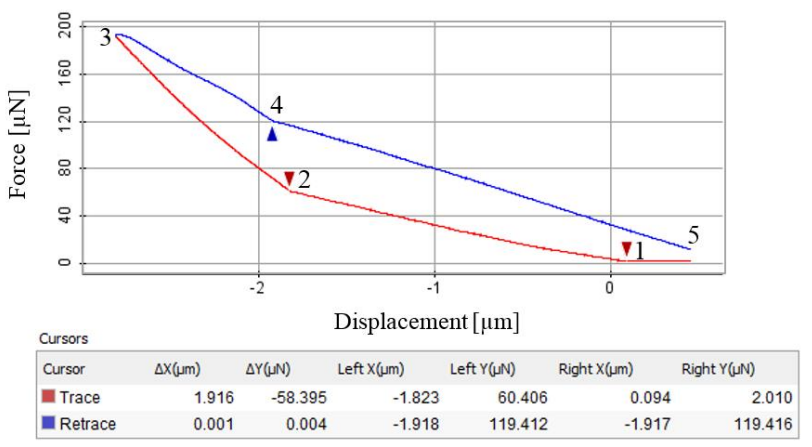

Fig. 3. AFM experimental curve at $20^{\circ} \mathrm{C}$ and $50 \% \mathrm{RH}$.

Firstly, the AFM probe comes in contact with the investigated sample in the midposition of central plate (Fig. 2). Secondly, the vertical displacement of the AFM probe 
together with the scanning head is controlled. The sample is fixed on the AFM table and the probe is mounted in the scanning head. In this step, the bending deflection of AFM probe is optically determined while the AFM probe bends together with the sample. The stiffness of the AFM probe is known. The detected deflection of AFM probe multiply with its stiffness give the force applied on structure.

Figure 3 presents the AFM experimental dependence between the vertical displacement of AFM probe and the applied force. There are two different curves, each of them with two slopes. The loading curve corresponds to the part 1-2-3 (Fig. 3) and the unloading is given by distance between 3 and 5 with an intermediate position in 4 . The first part of curve 1-2 corresponds to the bending of AFM probe together with investigated sample. The sample touches the substrate in position 2, after that is only the bending deflection of AFM probe. The unloading process starts in position 3, there is only unbending deflection of AFM probe between 3 and 4 , in position 4 the sample is detached from substrate and comes together with the AFM probe to the initial position 5. The first part 1-2 is used to determine the sample stiffness and the adhesion between the central plate and substrate can be observed in the position 4 on the experimental AFM curve. The difference between controlled vertical displacement of the AFM probe (and the scanning head) and the detected deflection of AFM probe gives the displacement of investigated sample. The applied force divided by the sample displacement means the sample stiffness. The tests are performed under different temperatures and for different humidity of the testing environment conditions. For accuracy measurements, the tests were repeated five times and the average results were considered.

\section{Temperature effect on the optical MEMS component response}

The temperature applied on the investigated structure additionally introduces a thermal stress gradient. The thermal gradient deforms the micromirror based on thermal dilatation of structure. Moreover, the modulus of elasticity decreases under the thermal relaxation effect of material with influence on the mechanical response of system [4]. The mechanical response of system depends on the out-of-the plane stiffness that is directly proportional with the modulus of elasticity of material. As a consequence, by increasing the operating temperature of structure the stiffness decreases as well as the mechanical response of micromirror.

Experimentally, the temperature is applied directly to the sample anchors between $20^{\circ} \mathrm{C}$ and $140^{\circ}$ by using a thermal controlled stage. After, the spectroscopy-in point of AFM is used to bend the flexible plate of micromirror and the stiffness is determined. Figure 4 presents the experimental out-of-the plane stiffness of the structure as a function of temperature.

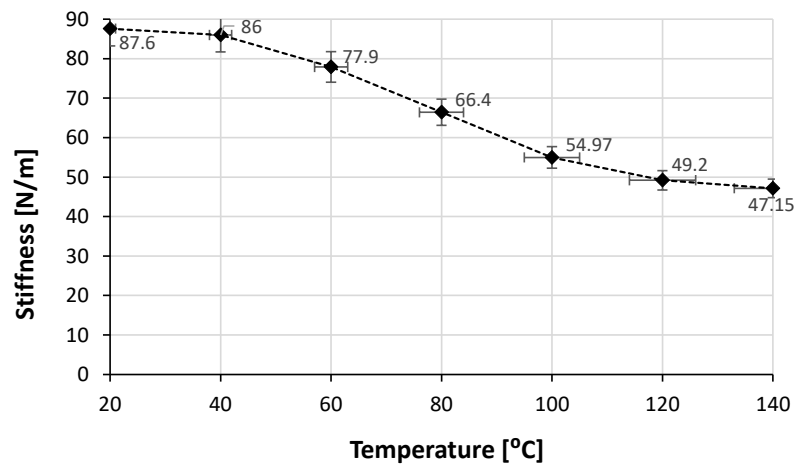

Fig. 4. Experimental out-of-the plane stiffness of micromirror versus temperature. 
The out-of-the plane stiffness decreases as temperature increases, respectively. A nonlinear dependence stiffness versus temperature is experimentally observed. The stiffness decreases with about $46 \%$ if the operating temperature increases from $20^{\circ} \mathrm{C}$ to $140^{\circ} \mathrm{C}$. At the beginning, a small decrease of the stiffness is determined, in the temperature range between $20^{\circ} \mathrm{C}$ and $40^{\circ} \mathrm{C}$. This is based on the relaxation of material provided by the residual stress appearing in the structure during fabrication process [6]. A strong dependence between stiffness and temperature is determined in the temperature range between $40^{\circ} \mathrm{C}$ and $140^{\circ} \mathrm{C}$ based on thermal relaxation of the modulus of elasticity of the material.

\section{Humidity influence on the optical MEMS components response}

In order, to determine the humidity effect on the behaviour of investigated micromirror, a humidity controlled chamber is used. The humidity is modified between $50 \% \mathrm{RH}$ and $90 \% \mathrm{RH}$. The humidity has not a significant influence on the micromirror stiffness. A small difference in stiffness of micromirror is experimentally determined if the humidity increases from $50 \%$ to $90 \%$ based on the damping effect given by the surrounding medium.

The spectroscopy-in-point of AFM is used to bend the central plate of micromirror until substrate under different controlled humidity. During unloading, the detachment of investigated micromirror from substrate (the position 4 from Fig. 3 ) is delayed due to the adhesion effect that gives a jump in the AFM probe deflection (Fig. 5). The jump size is directly proportional to the strength of adhesion between contacted surfaces. The tests performed on the adhesion between the flexible plate and substrate at room conditions $(50 \% \mathrm{RH})$ provides small adhesion force, around $4 \mathrm{nN}$ (Fig. 3). When the humidity is increased, the adhesion effect between contact surfaces increases significantly. The adhesion force is given by the difference between the cursors 4 ' and 4 "on the unloading AFM curve.

Figure 5 presents the adhesion force for two different humidities $(55 \% \mathrm{RH}$ and $90 \% \mathrm{RH})$. The value of the adhesion force is given by $\Delta \mathrm{Y}$ from the retrace values provided in the table under diagrams.

he relative humidity is step-by-step increasing between $50 \%$ and $90 \%$ and the adhesion force is measured. Figure 6 presents the humidity effect on the adhesion force between the investigated micromirror and its substrate. The adhesion effect increases as the medium humidity is increased, with a maximum measured force of $17.16 \mu \mathrm{N}$ at $90 \%$ RH.

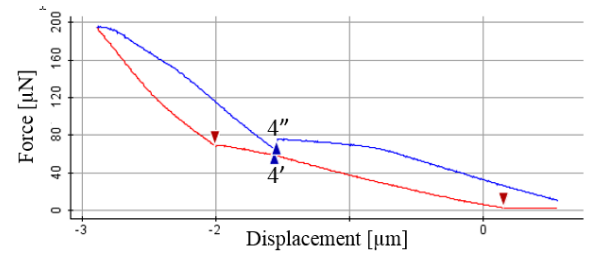

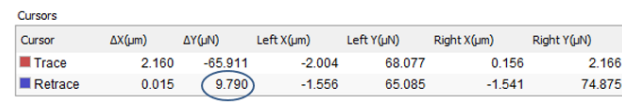

(a)

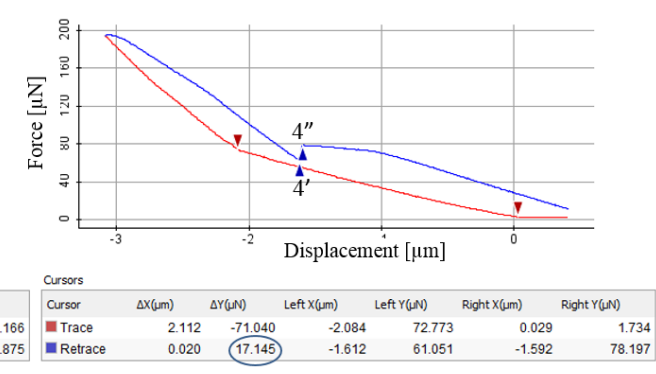

(b)

Fig. 5. Adhesion force between micromirror and substrate for: (a) $55 \% \mathrm{RH}$ and (b) $90 \% \mathrm{RH}$. 


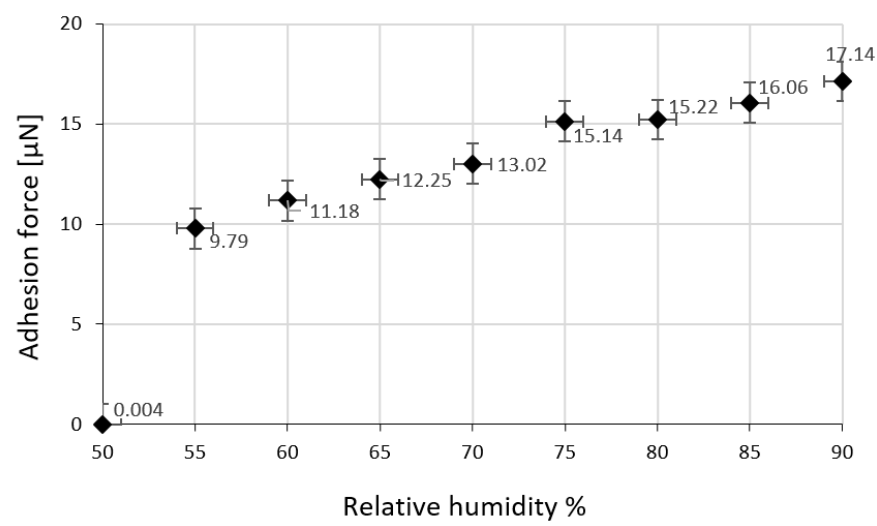

Fig. 6. Experimental adhesion force between micromirror and substrate versus relative humidity.

\section{Conclusions}

The environmental conditions change the mechanical and tribological behaviours of microcomponent from MEMS applications with influence on the accuracy in response and the system lifetime. In optical applications a temperature gradient occurs and changes the stiffness of flexible structures. A micromirror supported by rectangular hinges was chosen to investigate the environmental effect on the structure response. Out-of-the plane stiffness of microcomponent under a mechanical force was determined as well as the adhesion force. For adhesion analysis the flexible plate of microcomponent was deflected until substrate. The temperature was applied directly on the anchor using a controlled thermal stage. As the temperature increases, the stiffness decreases with influence on the system response (force and/or displacement). The stiffness decreases with about $46 \%$ if the testing temperature increases from $20^{\circ} \mathrm{C}$ to $140^{\circ} \mathrm{C}$. The temperature modifies the modulus of elasticity based on the thermal relaxation of material. Moreover, the stiffness has influence on adhesion effect based on the restorative force of microstructure from substrate.

There are some MEMS applications that operate in ambient conditions as the devices used in the environmental condition monitoring.

The humidity has a strong influence on adhesion effect based on the capillary forces. As the humidity increases, the capillarity is increased providing higher adhesion force between movable structure and substrate. Using a humidity controlled chamber, the relative humidity during testing was modified. For investigated MEMS structure, the adhesion force between the central plate and substrate significantly increases if the relative humidity is increased from $50 \% \mathrm{RH}$ to $90 \% \mathrm{RH}$.

The research work and the results presented in this paper are useful to MEMS designers in order to develop reliable MEMS structures operating under different environmental conditions.

This work was supported by a grant of the Romanian National Authority for Scientific Research and Innovation, CNCS/CCCDI UEFISCDI, project number PN-III-P2-2.1-PED-2016-1727, PNCDI III.

\section{References}

1. A. El-Fatatry, MEMS Aerospace Applications, MOEMS Applications (RTO-EN-AVT105 Lecture Series, NATO) (2015) 
2. I. Stanimirović, Z. Stanimirović, Optical MEMS for Telecommunications: Some Reliability Issues (In Advances in Micro/Nano Electromechanical Systems and Fabrication Technologies, InTech) (2013)

3. P. Schmitt, F. Pressecq, X. Lafontan, P. Pons, J. M. Nicot, C. Oudea, D. Estève, H. Camon, J.Y. Fourniols, MEMS behavioral simulation: a potential use for physics of failure $(\mathrm{PoF})$ modeling ( $4^{\text {th }}$ Round Table on Micro/Nano Technologies for Space) (2003)

4. M. Pustan, C. Dudescu, C. Birleanu, Phys. Statutus Solidi C 12, 1322-1327 (2015)

5. M. A. Tadayon, H. Sayyaadi, N. Jazar, J. of Physics: Conference Series 34, 89-94 (2006)

6. M. Pustan, V. Rochus, J.C. Golinval, Microsyst. Technol. 18, 247-256 (2012) 
